ENERGY AWARE ROUTING IN HIGH

CAPACITY OVERLAYS IN WIRELESS

SENSOR NETWORKS

By

SHARIFUL HASAN SHAIKOT

Bachelor of Science in Computer Science \& Information Technology Islamic University of Technology

Dhaka, Bangladesh

2002

Submitted to the Faculty of the

Graduate College of Oklahoma State University

in partial fulfillment of the requirements for the Degree of MASTER OF SCIENCE

May, 2007 
COPYRIGHT @

By

SHARIFUL HASAN SHAIKOT

May, 2007 


\section{ENERGY AWARE ROUTING IN HIGH CAPACITY OVERLAYS IN WIRELESS SENSOR NETWORKS}

Thesis Approved:

Venkatesh Sarangan

Thesis Advisor

Nohpill Park

Johnson Thomas

A. Gordon Emslie

Dean of the Graduate College 


\section{ACKNOWLEDGMENTS}

I would like to thank my advisor Dr. Venkatesh Sarangan for his invaluable suggestions, criticism, guidance and support during the course of my graduate study as well as for drafting the Master of Science thesis. The constructive and insightful discussions that I have had with him over the past few months has motivated me for developing new ideas for this work. I have always been benefited by his constant encouragement, enthusiasm and dedicated support for successful completion of my research work. It has been a privilege to have him as my mentor.

I would like to put on record the useful advices of Dr. Johnson Thomas and Dr. Nohpill Park of the Department of Computer Science and agreeing to be in my Graduate Committee. I am really grateful to them for their support. My sincere thanks and gratitude to the staff of the Computer Science Department for their cordial behavior and supportive attitude throughout my study in the said Department.

My Heartfelt gratitude and thanks to my family members, specially my parents who stood by me and motivated in all my endeavors throughout my career. Their constant supportive role and encouragement throughout my stay in the United States helped me to complete my Masters program successfully. I am also thankful to my two very loving sisters who constantly motivated me in my endeavors for higher studies. Last but not the least, I am grateful to the God for His blessings upon me and for His support in order to complete the Masters program in a timely fashion. 


\section{TABLE OF CONTENTS}

Chapter Page

1 INTRODUCTION 1

2 BACKGROUND: SENSOR AND SENSOR NETWORKS 4

2.1 The sensor model . . . . . . . . . . . . . . . . . . . . . . 4

2.2 Structure and organization of a wireless sensor network . . . . . . 5

2.3 MCDS Overview . . . . . . . . . . . . . . . 6

2.4 Shortest path . . . . . . . . . . . . . . . 7

3 RELATED WORK $\quad 8$

4 PROPOSED SCHEME $\quad 10$

4.1 Backbone construction Phase . . . . . . . . . . . . . . . 10

4.1.1 Finding MCDS in the Network . . . . . . . . . . . . 10

4.1.2 Finding SP in the Network . . . . . . . . . . . . . . . . 12

4.2 Routing phase . . . . . . . . . . . . . . . . . 12

4.3 Backbone Maintenance Phase . . . . . . . . . . . . . . . . . . . . 13

4.4 Energy consumption calculation . . . . . . . . . . . . . . . . . 14

5 PERFORMANCE EVALUATION $\quad 15$

5.1 Performance metrics . . . . . . . . . . . . . . . . . . 17

5.2 Simulation Results . . . . . . . . . . . . . . . . . 17

6 CONCLUSIONS $\quad 21$ 


\section{LIST OF TABLES}

Table

1.1 The energy consumption measurements of LUCENT IEEE 802.11 11 Mbps WaveLan PC Card . . . . . . . . . . . . . . . . . . . 3 


\section{LIST OF FIGURES}

Figure Page

1.1 A sample Wireless Sensor Network . . . . . . . . . . . . . . . . 2

2.1 A sample graph for MCDS . . . . . . . . . . . . . . . 6

5.1 A sample Topology used in the simulation run . . . . . . . . . . . . 16

5.2 Total Energy Consumption in the Network with respect to active time data generation rate for Frequent Events . . . . . . . . . . . . 18

5.3 Packet Delivery Fraction in the Network with respect to active time data generation rate for Frequent Events . . . . . . . . . . . . . . . 19

5.4 Total Energy Consumption in the Network with respect to active time data generation rate for Infrequent Events . . . . . . . . . . .

5.5 Packet Delivery Fraction in the Network with respect to active time data generation rate for Infrequent Events . . . . . . . . . . . . 19

5.6 Total Energy Consumption in the Network with respect to active time data generation rate for Frequent Events . . . . . . . . . . . . . 20

5.7 Total Energy Consumption in the Network with respect to active time data generation rate for Infrequent Events . . . . . . . . . . . . 20 


\section{CHAPTER 1}

\section{INTRODUCTION}

A wireless sensor network consists of one or multiple data centers called the physical sinks and many low-cost and low-powered sensor devices, called sensor nodes as shown in figure 1.1. Each sensor node has the ability to sense data, process data, and share this data with others via radio transceivers. The sink node, equipped with a database system, sends queries or control commands to sensor nodes and collects information from sensors. The communication between the sink and sensor nodes relies on relaying by intermediate sensor nodes [1]. Energy consumption in wireless sensor networks (WSNs) is universally recognized as an important issue. As the sensor nodes are microelectronic devices, they can only be equipped with a limited power source. The sources of power consumption are communication and computation, with communication often being the chief power consumer.

In order to control congestion in the sensor networks and reduce the packet loss rate, researchers have proposed the idea of dispersing virtual sinks (VSs) in the sensing field [2]. The VSs usually have two different interfaces - a low power, low bandwidth, short range mote interface to communicate with the sensors and a high power, high bandwidth, long range 802.11 interface to communicate with other VSs and the sink. In other words, the 802.11 links in the VSs form a high capacity overlay on top of the sensor network. As the VSs are battery powered, it is important to reduce the energy consumption in them too. In this thesis work, we focus on reducing energy consumption in the VSs.

Feeney et al. [3] have documented the actual power drawn by a popular 802.11 


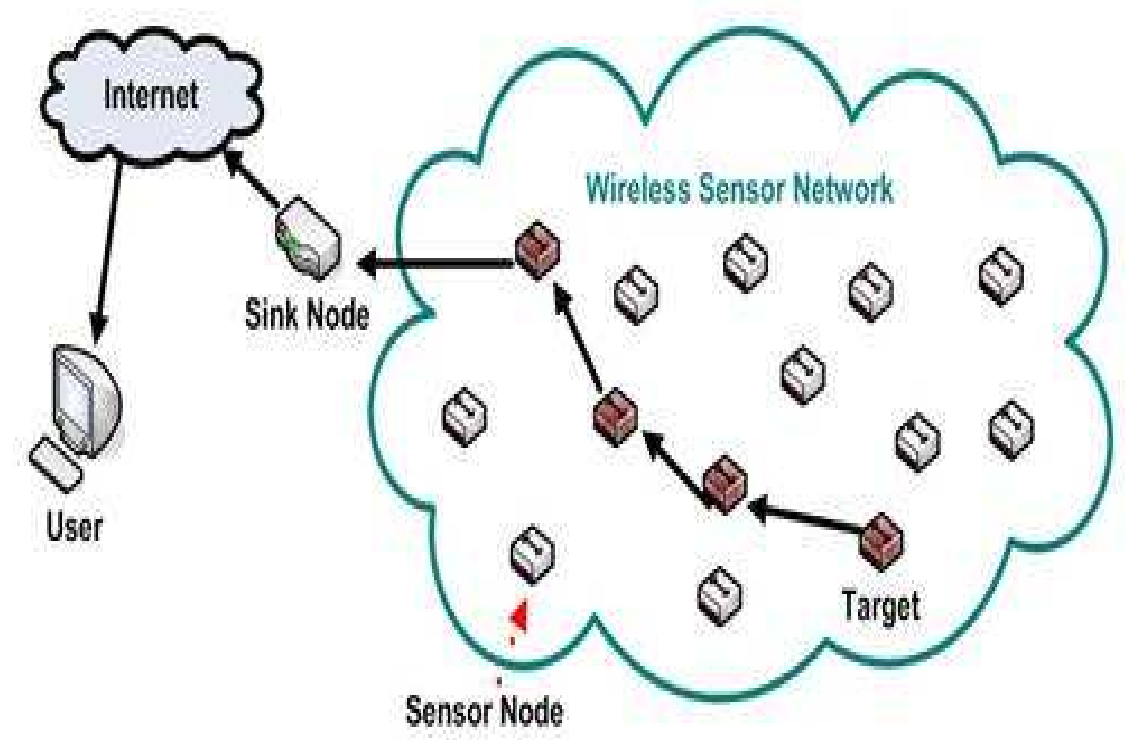

Figure 1.1: A sample Wireless Sensor Network

network interface card in the four possible modes as shown in Table 1.1. Receive and idle mode require similar power, and transmit mode requires slightly greater power. Sleep mode requires more than an order of magnitude less power than idle mode. These measurements show that the network interface expends similar energy, whether it is just listening or actually receiving data. Therefore, leaving the 802.11 transceiver always on (i.e. idle listening) for long periods of time can lead to significant energy consumption and thus impact the longevity of the network [4].

A good way to save energy is to turn off the VS when they are not in use. In the absence of topology control, data from a VS is usually routed along the Shortest Path (SP) route in the overlay. However, if only a few of the VS nodes are sending data, routing along the SP may require some additional VS nodes to be turned on just for the relaying purpose which otherwise could be turned off. Since the link bandwidth is high in 802.11 ( Mbps) and the sensory data generation rate is low ( $\tilde{K} b p s)$ in comparison to the bandwidth, a high idle-mode energy cost may be incurred in the relaying VS nodes. In this thesis work, we explore the idea of using minimum connected dominating set (MCDS) based routes, since more energy can be 


\begin{tabular}{|c|c|c|}
\hline Mode & Energy Consumption & Power Consumption \\
\hline Transmit & $\begin{array}{c}0.48^{*} 10^{-6} \text { joule/byte } \\
+431^{*} 10^{-6} \text { joule }\end{array}$ & $1346.16 \mathrm{~mW}$ \\
\hline Receive & $\begin{array}{c}0.12^{*} 10^{-6} \text { joule } / \text { byte } \\
+316^{*} 10^{-6} \text { joule }\end{array}$ & $900.6 \mathrm{~mW}$ \\
\hline Idle & 0.739 joule $/ \mathrm{sec}$ & $739.44 \mathrm{~mW}$ \\
\hline Sleep & - & $66.36 \mathrm{~mW}$ \\
\hline
\end{tabular}

Table 1.1: The energy consumption measurements of LUCENT IEEE $802.1111 \mathrm{Mbps}$ WaveLan PC Card

saved by switching the non-dominator VSs to sleep mode and by funneling all the data through the MCDS nodes. We propose an energy-aware routing scheme that considers both the SP route and the MCDS nodes to discover a path along the VS network to the physical sink. Performance evaluation of the routing scheme shows a notable reduction in the overall energy consumption in the network with respect to SP routing while simultaneously maintaining an acceptable packet delivery rate. 


\section{CHAPTER 2}

\section{BACKGROUND: SENSOR AND SENSOR NETWORKS}

The main goal of this section is to review [5] a number of common assumptions about sensors and the resulting wireless sensor networks that will be used throughout the remainder of the paper.

\subsection{The sensor model}

We assume a sensor to be a device that possesses three basic capabilities; sensory, computation, and communication. A sensory capability is necessary to acquire data from the environment. A communication capability is necessary for sending (receiving) aggregated data and control information to (from) other sensors or the physical sink. A computational capability is necessary for aggregating data, processing control information, and managing both sensory and communication activity. It is widely believed that the following features will impact the design of sensor network algorithms:

- Sensor nodes are limited in power, computational capability and memory

- Sensor networks are highly dynamic (in tasks, environment settings, and network topology)

- Large number of sensor nodes are densely deployed

- Sensor nodes must work unattended

- Sensor nodes are prone to failure

- Sensor nodes work cooperatively 
- Sensor networks are application-specific

The sensor nodes are likely to be battery powered, and it is often very difficult to change or recharge batteries for these sensors. For each sensor, communication consumes much more power than computing. In addition, research shows that in a low-energy radio network, the energy consumed by receiving and listening (attempting to receive) message is of the same order of magnitude as transmitting them. In sensor networks, leaving the transceiver on (i.e. idle listening) for long periods will be the major factor that impacts the longevity of the network [4].

\subsection{Structure and organization of a wireless sensor network}

We assume that individual sensor nodes operate subject to two fundamental constraints. First, each sensor has a nonrenewable power budget. Second, each sensor has a modest transmission range, perhaps a few meters. The latter constraint implies that outbound messages sent by a sensor can reach only the sensors in its proximity, typically a small fraction of the sensors in the entire network. As a consequence, the sensor network must be multi-hop and only a limited number of the sensor nodes count the physical sink among their one-hop neighbors. We assume that the sensor network is connected to the outside world (e.g. point of command and control, the Internet, etc.) through a physical sink node. The physical sink has a full range of computational capabilities, can send long-range directional broadcasts to all sensors, can receive messages from nearby sensors, and has a steady power supply. The sink is a single point of failure in this model. Importantly, the sink is also in charge of performing any necessary training and maintenance operations involving the sensor network. The limited power budget of individual nodes mandates the design of energy-efficient data gathering, fusing, and communication protocols. One of the major concerns in designing protocols for wireless sensor networks is scalability. We expect there are enough nodes in the sensor network and these nodes are densely deployed. Only a 


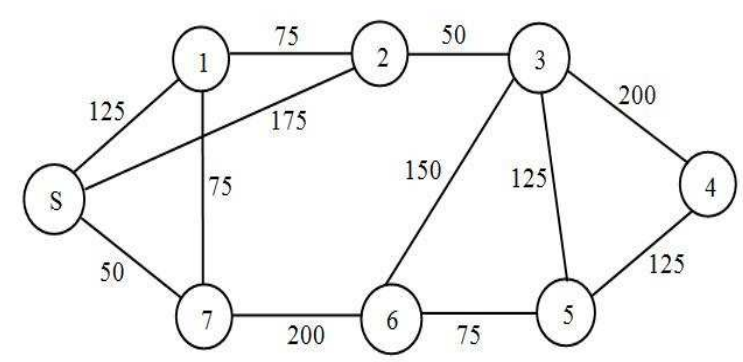

MCDS\#1: $\{1,2,3\}$

MCDS\#2: $\{2,3,6\}$

MCDS\#3: $\{3,6,7\}$

Figure 2.1: A sample graph for MCDS

small set of them is needed to work (e.g. sensing, transmitting) at any moment.

\subsection{MCDS Overview}

In order to represent the wireless sensor network we can use a simple graph $G=$ $(V ; E)$, where $V$ represents a set of wireless mobile nodes and $E$ represents a set of edges. An edge between node pairs $\{v, u\}$ indicates that both nodes $v$ and $u$ are within each other's wireless transmitter ranges.

To simplify our discussion, we assume all mobile nodes are homogeneous, i.e., their transmitter ranges are the same. Thus the corresponding graph will be an undirected graph [6]. Dominating-set-based routing $[6,7]$ is based on the concept of dominating set in graph theory [8]. This concept of routing is valid only for networks, which can be represented by connected graphs. So, in this work we will consider only connected graphs. A subset of the vertices of a graph is a dominating set if every vertex not in the subset is adjacent to at least one vertex in the subset $[9]$. In a graph $G=(V ; E)$ a node $v_{i}$ is considered to be adjacent to another node $v_{j}$ if there exists an edge between $v_{i}$ and $v_{j}$. Given a simple undirected graph $G=(V ; E)$ with the set of vertices $V$ and the set of edges $E$, a dominating set (DS) is a set $D \subseteq V$ such that each vertex in $V \backslash D$ is adjacent to at least one vertex in D. A connected dominating set (CDS) is a dominating set, which is also a connected sub graph of the original graph $G$ and 
is a set of node, that is, there is a path between any two nodes in CDS that does not use nodes that are not in CDS. A minimum connected dominating set (MCDS) is a connected dominating set such that removal of any node from that set makes it a non-dominating set.

In figure 2.1, the possible MCDS are $\{1,2,3\},\{2,3,6\}$ and $\{3,6,7\}$. Main advantage of this approach is that searching space for a route is reduced to the nodes in the dominating set. As long as changes in network topology do not affect this sub network there is no need to recalculate routing tables.

\subsection{Shortest path}

We use the Single source shortest path to find out the shortest path from the Physical sink to all the Virtual Sinks in the wireless sensor networks. We use the distributed Bellman-Ford algorithm to calculate the single source shortest path. The "number of hops" is the metric in the calculation of the shortest path. 


\section{CHAPTER 3}

\section{RELATED WORK}

A virtual sink may have to operate for a relatively long duration on a tiny battery and thus energy efficiency becomes a major concern.

A variety of "power-aware" routing protocols have been proposed to address this problem. In one school of thought [10]-[11], the traditional Shortest Path First strategy is replaced by Least Energy First routing, i.e., a multi-hop route is preferred to a single-hop one if only multiple short-distance relays cost less energy than a single long-distance transmission. For example, "Minimum Transmission Energy" (MTE) routing [12], [11] was proposed in place of traditional "minimum hops routing". Another school of thought is that nodes are clustered so that a hierarchy is formed [13]$[14]$.

Wu et al proposed [15] a new algorithm, for power aware minimum connected dominating set. This does not suggest a dynamic selection scheme, where if energy levels of the dominating nodes fall below a threshold level, an alternate connected dominating set (if it exists at all) can be formed from the nodes whose energy levels are above the threshold energy level. Moreover, if we assign random energy levels to the nodes of a connected graph (with some nodes having zero energy levels) the algorithm in [15] may form a minimum connected dominating set with nodes having zero energy level. T. Acharya et al. [9] proposed a new scheme (based on the distributed implementation of the heuristic algorithm proposed in [16]) to construct a power aware minimum connected dominating set which solves the problem of [15].

Another class of energy-saving schemes are the wakeup schemes [17]-[18] that ex- 
ploit the temporal potential in energy saving by turning off radios when not needed and provide some mechanisms to wake the radio up when necessary. Wakeup schemes have great potential in energy saving for sensor networks where events occur infrequently. When events occur frequently, the proposed wakeup schemes, e.g., [17], [19][20], encounter critical tradeoffs between energy saving and wakeup delay. Xue et al [21] proposed another new wakeup scheme to reduce the end-to-end delay, but unfortunately the end-to-end delay still exists.

We explore an alternative, possibly complementary, approach to the above schemes by turning off as many virtual sinks as possible in the overlay. Since the sensor nodes generate only a few Kbps of data and the bandwidth of the overlay links is few Mbps, we can reduce substantial amounts of idle-wastage by intelligently turning off some virtual sinks. Although turning off some nodes may decrease the capacity of adhoc networks, it does not concern us because of the fact that the data generation rate in the sensor nodes is quite small. The overlay therefore is never pushed to its limits. Performance evaluation of the proposed scheme shows a notable reduction in the overall energy consumption while maintaining an acceptable packet delivery rate. 


\section{CHAPTER 4}

\section{PROPOSED SCHEME}

The central idea of our proposed scheme is to find out a path from the virtual sink to the physical sink such that this allows us to turn off more virtual sinks and thus save energy. In order to achieve this, we exploit the concept of both the MCDS and the SP. The scheme that we proposed has 3 phases. First of all, the Backbone construction phase, then the Routing phase and last of all the Backbone maintenance phase. The proposed scheme is detailed below:

\subsection{Backbone construction Phase}

In backbone construction phase, there are 2 sub phases. In the first sub-phase, the Physical Sink (PS) generates the MCDS based routes for the VSs. In the second phase, the PS generates the Shortest path (SP) routes from all the Virtual Sinks(VS) to the PS.

\subsubsection{Finding MCDS in the Network}

A Threshold Energy Level $E_{t h}$ is set in order to determine the eligibility of a node to become a dominator. Since a dominating node is responsible for forwarding packets of other nodes in the network in addition to its own packets, it must have adequate energy level to be able to perform both kinds of tasks. In order to be eligible for becoming a dominating node the energy level of the node must be greater than $E_{t h}$. A power aware (energy efficient) distributed Minimum Connected Dominating Set (MCDS) algorithm [9] is used to build the backbone. We assume that the Physical 
Sink (PS) node has the information about the overlay topology. This can be obtained through a one-time exchange of Hello messages. Based on this information, the MCDS nodes are selected by the PS and this information is disseminated to all the VS nodes. Each non-MCDS VS chooses a MCDS neighbor as its downstream node in the path to the PS. Incase a VS has two dominators in 1-hop distance, the VS will choose the dominator with smaller distance. The PS has a MCDS table in which the PS stores the information about multiple MCDS (if any) in the network. The multiple MCDS has the priority key in the MCDS table and the MCDS with the highest priority is selected for routing. In case of presence of multiple MCDS in the network, the criteria used by the PS for selecting a MCDS is as follows:

i Select the MCDS with the smallest cardinality.

ii If the cardinality of MCDS is same for all MCDSs, then select the MCDS that has the most MCDS nodes with 1-hop link to the PS.

iii If the number of MCDS node with 1-hop link to PS is same for all MCDSs, then select the MCDS which has the smallest 1-hop distance with the PS.

iv If above three criteria can not resolve the tie, then calculate the average distance per neighbor for the MCDS nodes and select the MCDS with the smallest average distance per neighbor. To calculate the average distance per neighbor, find out the number of neighbors of each MCDS node in the MCDS and sum up the distances to all the neighbors for each MCDS node. Then divide the total distance by total number of neighbors. Assume, Average distance per neighbor $D_{\text {avg. }}$. The equation is as follows:

$$
D_{a v g}=\frac{1}{N} \sum_{i \in M C D S \text { and } i, j \in E} d_{i, j}
$$

where $N=\sum_{i \in M C D S}$ neighbor $_{i}$ 
We use distance as a tie-breaking criteria since nodes that are closer to each other generally have a better quality link between them than otherwise.

\subsubsection{Finding SP in the Network}

The PS already has the topology of the entire overlay. With this information, the Shortest Path (SP) from each VS to the PS can be calculated.

\subsection{Routing phase}

During routing phase, the communication is two-way, from the PS to the VS as well as from the VS to the PS.

- The VS that wants to send data performs following tasks:

i Check if the PS is 1-hop neighbor,

(a) If Yes, send the data packet/message packet directly to the PS If No, notify its dominator about its intention or to next hop dominator node incase the sending node itself is a dominator node

- The dominator performs following tasks upon receiving the data packet/ message packet:

i If the message is from Physical Sink(special bit is ON),

(a) If the destination candidate node is non-dominator VS node then

i. If the destination candidate non-dominator VS node is 1-hop neighbor, then Send the message/packet directly to the candidate node Else Send the message/packet to its next hop dominator Else If destination candidate node is dominator VS node then Send the message to the next hop dominator. The candidate node will look up 
the recipient address and if the message/packet is destined for itself, the candidate node will stop forwarding the packet

ii If the message is from VS(special bit is OFF),

(a) If the PS is 1-hop neighbor, Send the message directly to the PS Else notify its next hop dominator about the message/data packet

Eventually, the PS or candidate VS node will get the message/packet. The PS has MCDS information of the network and also SP information for that candidate VS node. The PS calculates $E_{M C D S}$ and $E_{S P}$ for the candidate node where $E_{i}=$ Energy consumed under method $i$. The PS sends the routing path information to the candidate node using the path decided by the PS (either MCDS or SP). The command packet travelled from the PS to the candidate node contains the information of the nodes of the routing path. The PS performs the following task for selecting the routing path

i If $E_{M C D S}>E_{S P}$, notify the candidate node the SP as routing path

Else notify the candidate node the MCDS path as routing path

\subsection{Backbone Maintenance Phase}

The PS periodically evaluates the eligibility of the dominators by checking their energy levels with the threshold. The dominator nodes send periodic Hello message to its next-hop dominator. This Hello message includes the information about the status of the sending node's residual energy levels. Such messages are forwarded to the PS so that the PS can have information regarding the residual energy in all the dominators. If energy level of any MCDS node is about to fall below the $E_{t h}$ then the PS picks up the next available MCDS from its MCDS table for constructing the alternative backbone. 


\subsection{Energy consumption calculation}

In this section, we describe the details of calculation of Energy consumption. The equations for calculating the energy consumption in MCDS method is $E_{M C D S}$ and Shortest Path method is $E_{S P}$ where $E_{i}=$ Energy consumed if communication occurs in $i$ method. We assume, $M C D S N=$ MCDS Path Node, $S P N=$ Shortest Path Node and $N M C D S S P=$ nodes $\notin M C D S$ path and nodes $\in S P$

$$
\begin{gathered}
E_{M C D S}=\sum_{M C D S N} E_{T X}+\sum_{M C D S N} E_{R X} \\
E_{S P}=\sum_{S P N} E_{T X}+\sum_{S P N} E_{R X}+\sum_{N M C D S S P}\left\{1-\frac{T X+R X}{B W}\right\} E_{\text {idle }}
\end{gathered}
$$

where $E_{T X}$ is the energy consumed during transmission, $E_{R X}$ is the energy consumed during reception, $E_{\text {idle }}$ is the energy consumed in idle mode, $T X=$ amount of data transmitted in bits, $R X=$ amount of data received in bits, $B W=$ Bandwidth of the WNIC. Since MCDS nodes will always remain turned on in order to maintain the connectivity of the network, we ignore the idle mode energy consumption of MCDS method. However, it may happen that in Shortest Path(SP) method, some nodes that are not in MCDS but in SP need to be turned on in order to transmit the packet to the Physical sink. Therefore, we consider the idle mode energy consumption in SP method by including the term $\sum_{N M C D S S P}\left\{1-\frac{T X+R X}{B W}\right\} E_{\text {idle }}$ in equation 4.3. 


\section{CHAPTER 5}

\section{PERFORMANCE EVALUATION}

In this section, we report on extensive simulation-based studies on the performance impacts of MCDS only scheme, SP only scheme and our proposed scheme in the ns-2 [22] network simulator using the CMU wireless extensions [23]. The short description of the schemes are as follows:

i MCDS only Scheme: In this scheme, the nodes in the MCDS are remained turned on and the message/data packets are routed along the MCDS nodes to the Physical Sink.

ii SP only Scheme: In this scheme, the nodes in the Shortest Path are remained turned on and the message/data packets are routed along the Shortest Path to the Physical Sink.

iii Our Proposed Scheme: In this scheme, we find out the energy aware routing path based on the energy consumption $E_{M C D S}$ and $E_{S P}$ as shown in equation (4.2) and (4.3). The nodes of the selected routing path are remained turned on and the message/data packets are routed along that path to the Physical Sink.

We use simulation results to evaluate all the schemes mentioned above in terms of energy consumption and packet delivery fraction(PDF). To model a Virtual Sink (VS) node we add support for a second long-range radio interface that has a transmission range of $250 \mathrm{~m}$. The primary low-power radio used in our simulations is configured to have a $40 \mathrm{~m}$ transmission range to model a typical sensor node. In most of our simulations, we use a fixed workload that consists of eight sources and one physical 


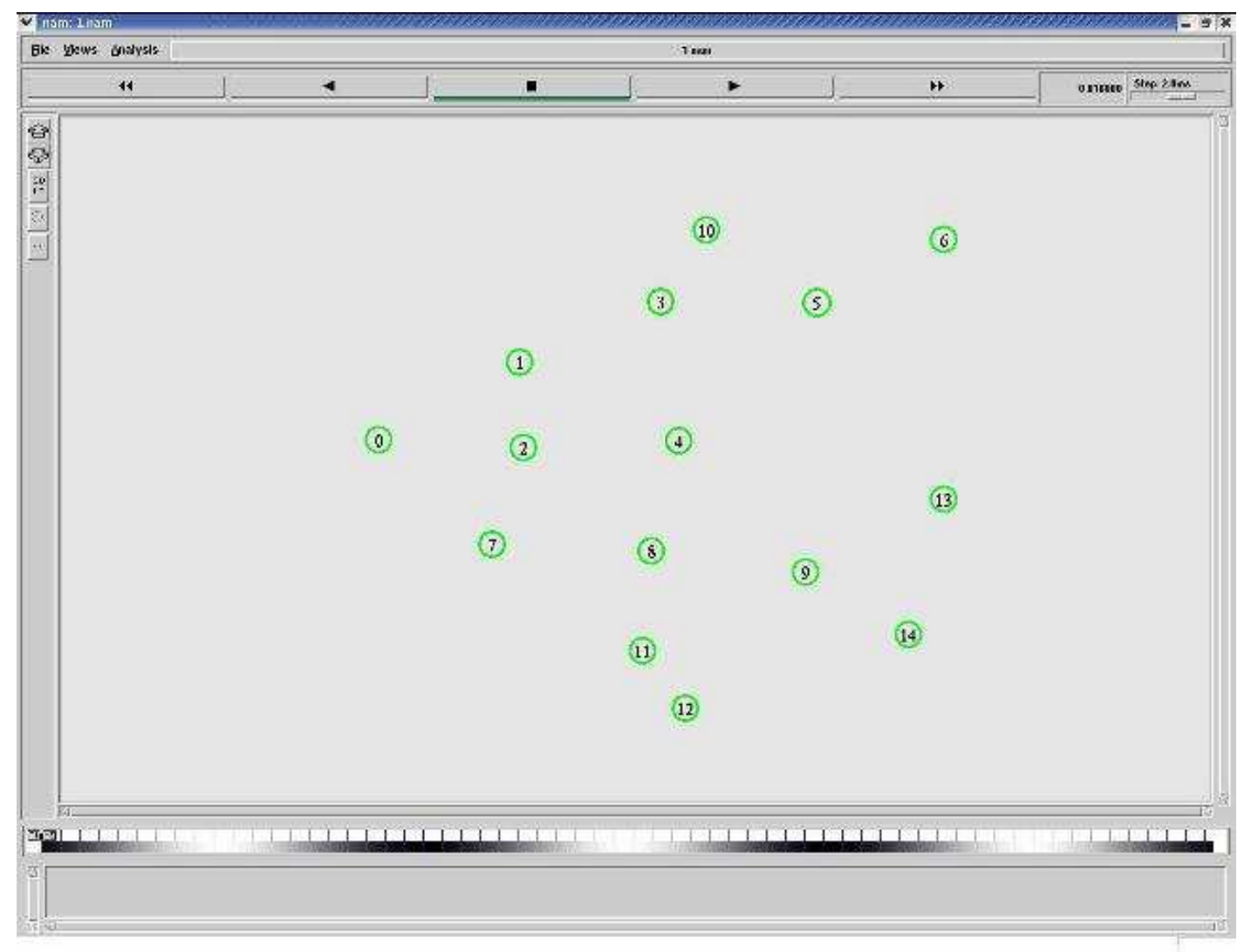

Figure 5.1: A sample Topology used in the simulation run

sink. The traffic for our simulation studies consists of Constant Bit Rate (CBR) sources over UDP flows. In UDP flows, packets are sent by the sensor nodes at regular intervals. The packet sending nodes send a CBR flow to the physical sink and each CBR flow sends $10 \mathrm{Kbps}, 25 \mathrm{Kbps}, 40 \mathrm{Kbps}, 55 \mathrm{Kbps}, 70 \mathrm{Kbps}$ and $85 \mathrm{Kbps}$ of traffic. In all our experiments, we use random topologies with different network sizes having up to 15 nodes randomly distributed on a square region, to study the effects of various schemes on energy consumption and packet delivery fraction (PDF).

In this section, we discuss in detail results from one representative topology, where 15 nodes were distributed over a 500X500 unit grid as shown in figure 5.1. The initial energy for each node is set to 1000 joules and the simulations are run for 900 simulated seconds. The radio model uses characteristics similar to a commercial radio interface, Lucent's WaveLAN. WaveLAN is modelled with a nominal bit rate of $2 \mathrm{Mb} / \mathrm{s}, 11$ 
$\mathrm{Mb} / \mathrm{s}, 48 \mathrm{Mb} / \mathrm{s}, 54 \mathrm{Mb} / \mathrm{s}$ and $108 \mathrm{Mb} / \mathrm{s}$.

\subsection{Performance metrics}

We define the following metrics to analyze the performance of our proposed scheme.

- Energy consumption of the network: The total amount of energy consumed by all the nodes in the network in transmitting, receiving and idle mode.

- Packet Delivery Fraction(PDF): The ratio of the data packets delivered to the destinations to those generated by the CBR sources.

$P D F=\frac{\text { received packets }}{\text { sent packets }} * 100$

We use these metrics to evaluate and quantify the benefits of using our proposed scheme under different scenarios and configurations in the following section.

\subsection{Simulation Results}

In our simulation, we use following two events.

- Frequent Events: The nodes send data frequently from the starting to the end of the simulation run.

- In-frequent Events: The nodes send data randomly during the simulation run.

For each event, the following two scenarios have been simulated.

- Different Data Generation Rate: In this scenario, the nodes are sending data at different rates in Kbps.

- Different WNIC Bandwidth: In this scenario, the data generation rate is kept constant (e.g. $25 \mathrm{Kbps}$ ) but the bandwidth of the WNIC has been varied. 


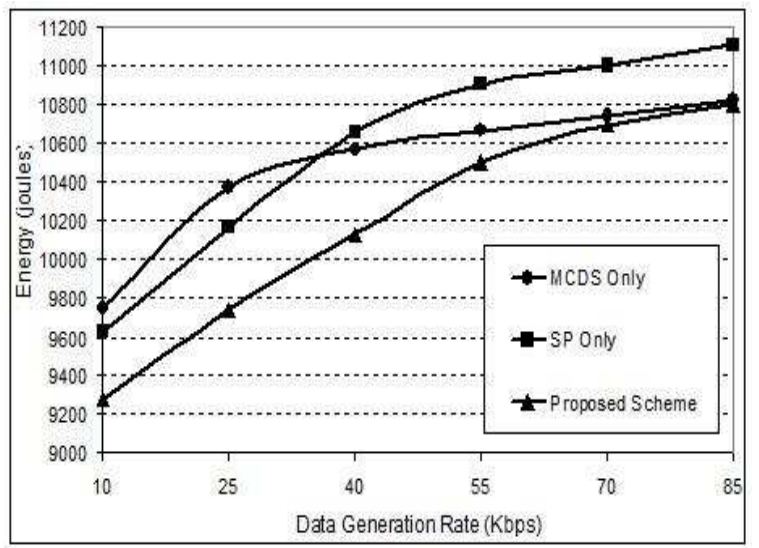

Figure 5.2: Total Energy Consumption in the Network with respect to active time data generation rate for Frequent Events

Figure 5.2 plots the total energy consumed in the network by a UDP flow and the different active time data generation rate by the sending node for frequent events. Figure 5.2 shows that the total energy consumed in the network is least in our proposed scheme. Neither the MCDS only scheme nor the SP only scheme alone performs better than our proposed scheme. Compared to MCDS only and SP only scheme, our proposed scheme shows an approximately 4\%-6\% improvement. This is because in our proposed scheme, we select the routing path by considering both $E_{M C D S}$ and $E_{S P}$ and the path in which less energy is consumed is chosen. Thus, our proposed scheme demonstrates the energy efficiency compared to the MCDS only or SP only scheme.

Figure 5.3 shows the Packet Delivery Fraction(PDF) in the network for three different schemes. From these results, we find that our proposed scheme successfully delivers most number of packets to the physical sink compared to other two schemes. In other word, the packet loss rate is least in our proposed scheme. Hence, performance of our proposed scheme is better than that of other two schemes.

We also run the simulation for infrequent events. Figure 5.4 and 5.5 clearly shows that our proposed scheme also performs better than the MCDS only scheme and SP Only scheme for the infrequent events scenario. 


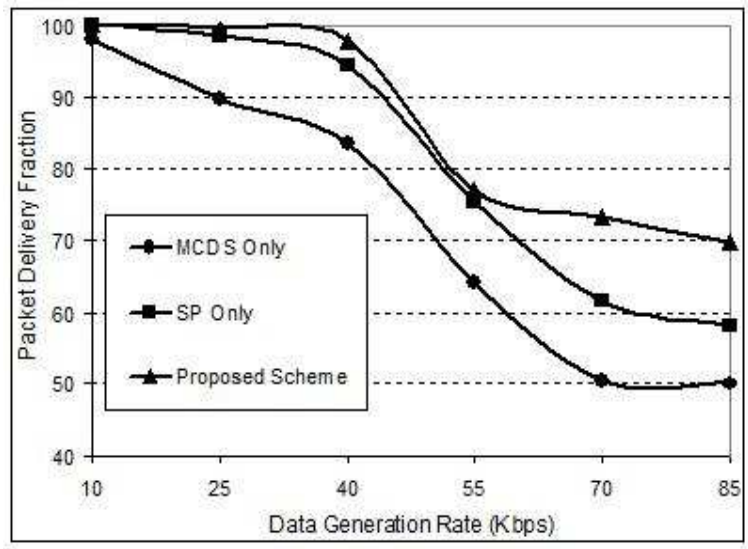

Figure 5.3: Packet Delivery Fraction in the Network with respect to active time data generation rate for Frequent Events

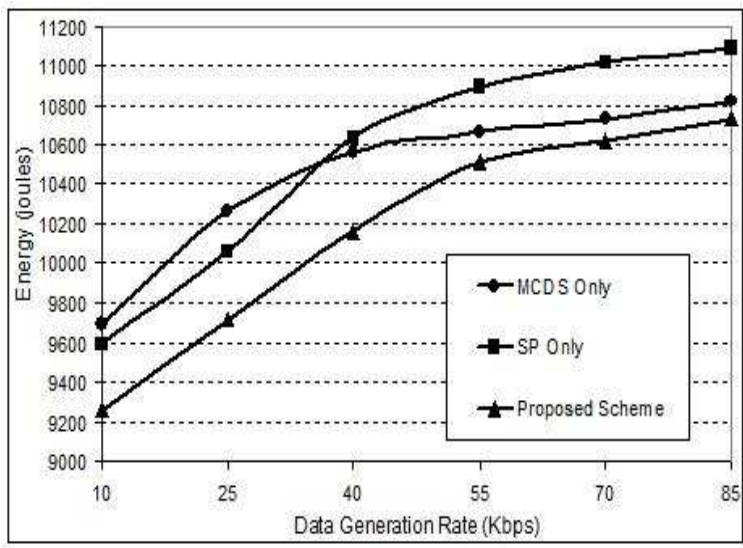

Figure 5.4: Total Energy Consumption in the Network with respect to active time data generation rate for Infrequent Events

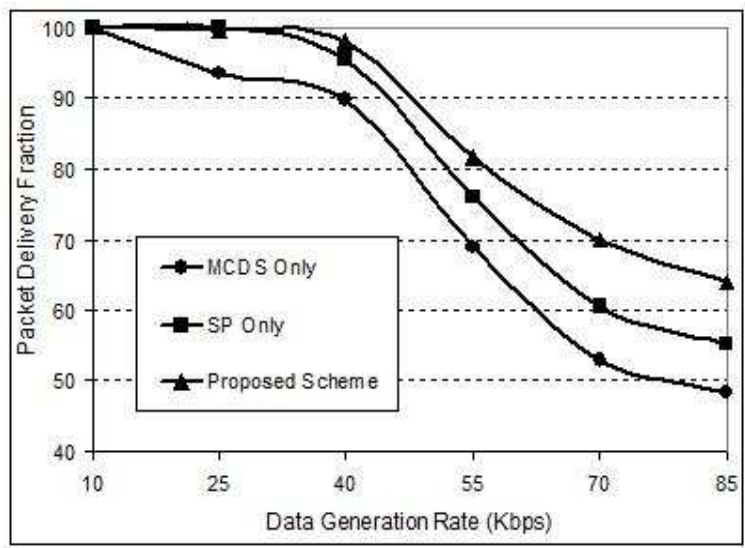

Figure 5.5: Packet Delivery Fraction in the Network with respect to active time data generation rate for Infrequent Events 


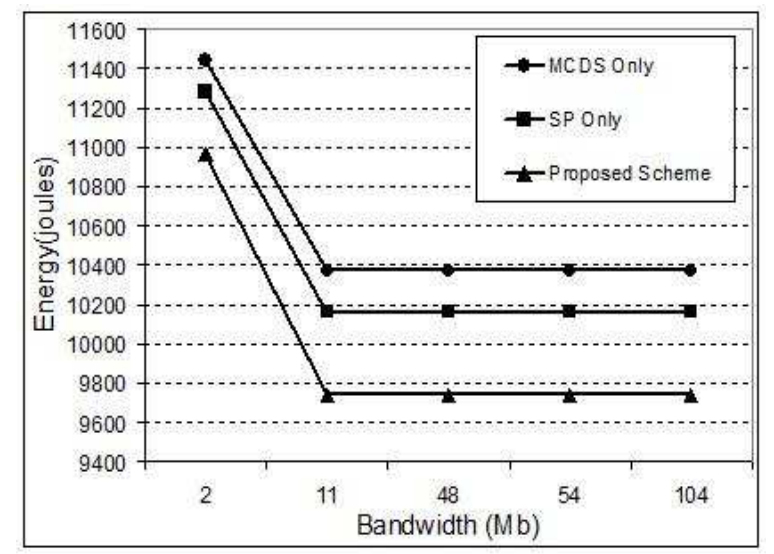

Figure 5.6: Total Energy Consumption in the Network with respect to active time data generation rate for Frequent Events

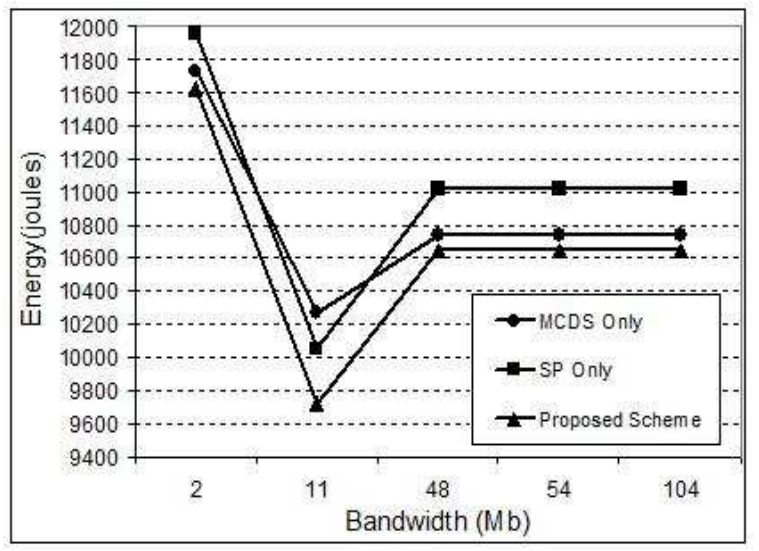

Figure 5.7: Total Energy Consumption in the Network with respect to active time data generation rate for Infrequent Events

We run our simulation in different WNIC bandwidth scenario for both frequent and infrequent events. Figure 5.6 plots the total energy consumed in the network by a UDP flow and the different bandwidth of the WNIC for the frequent events. The data sending rate is kept constant (e.g. $25 \mathrm{Kbps}$ ). From the figure 5.6 we find that our proposed scheme consumes least energy in the network in all different WNIC bandwidth compared to other schemes. From the figure 5.7 we find that our proposed scheme outperforms the MCDS only scheme and SP Only scheme for the infrequent events scenario. 


\section{CHAPTER 6}

\section{CONCLUSIONS}

Energy consumption in wireless sensor network is universally recognized as an important issue. Thus energy saving is a critical task for sensor networks.

In this work, we have presented a scheme for selecting an energy aware path from Virtual Sinks (VS) to the Physical Sink(PS) in wireless sensor networks. The proposed scheme reduces the energy consumption by turning off as many VS possible in the network. An energy aware path is constructed by exploiting both the minimum connected dominating set (MCDS) and the single source shortest path (SP) in the network. Simulation results show that if both the MCDS and SP are considered in

order to find out an energy aware path, considerable amount of energy can be saved in the network while maintaining an acceptable packet delivery rate. 


\section{BIBLIOGRAPHY}

[1] e. a. Zhang, W., "Energy-aware location-aided multicast routing in sensor networks," in Proceedings WCNM, pp. 901-904, November 2005.

[2] e. a. Chieh-Yih Wan, "Siphon: Overload traffic management using multi-radio virtual sinks in sensor networks," in SenSys'05, November 2-4 2005.

[3] M. N. Laura Marie Feeney, "Investigating the energy consumption of a wireless network interface in an ad hoc networking environment," IEEE INFOCOM, 2001.

[4] Y. S. I.F. Akyildiz, W. Su and E. Cayirci, "Wireless sensor networks: A survey," Computer Networks, 38(4), pp. 393-422, 2002.

[5] Q. X. S. Olariu and A. Y. Zomaya, "An energy-efficient self-organization protocol for wireless sensor networks," in ISSNIP, 2004.

[6] J. Wu and H. Li, "On calculating connected dominating set for efficient routing in ad hoc wireless networks," in in Proc. Of the 3rd Int'l Workshop on Discrete Algorithms and Methods for Mobile Computing and Commun., pp. 7-14, 1999.

[7] B. Das and V. Bhargavan, "Routing in ad-hoc networks using minimum connected dominating sets.," in IEEE International Conference on Communications (ICC'97)., June 1997.

[8] T. et al., Fundamentals of Domination in Graphs. Marcel Dekker, Inc. A Siries of Monographs and Text books, 1998. 
[9] R. R. T. Acharya, "Distributed algorithm for power aware minimum connected dominating set for routing in wireless ad hoc network," in the 2005 International Conference on Parallel Processing Workshops (ICPPW'05), 2005.

[10] S.-H. C. N. I. E. S. A. S. A. W. R. Min, M. Bhardwaj and A. Chandrakasan, "Energy-centric enabling technologies for wireless sensor networks," IEEE Wireless Commun. Mag., vol. 9, pp. 28-39, August 2002.

[11] M. Ettus, "System capacity, latency and power consumption in multihoprouted ss-cdma wireless networks," in in Proc. Radio and Wireless Conf. (RAWCON'98), (Colorado Springs, CO), pp. 55-58, Aug. 1998.

[12] T. Shepard, "A channel access scheme for large dense packet radio networks," in Proc. ACM SIGCOMM, (Stanford, CA), pp. 219-230, Aug. 1996.

[13] R. Ramanathan and M. Steenstrup, "Hierarchically-organized, multihop mobile wireless networks for quality-of-service support,," Mobile Networks Appl., vol. 3, no. 1, pp. 101-119, 1998.

[14] N. H. V. et al., "A cluster-based approach for routing in dynamic networks," in ACM Comput. Commun. Rev., vol. 17, Apr. 1997.

[15] M. G. J.Wu, F. Dai and I. Stojmenovic, "On calculating power-aware connected dominating sets for efficient routing in ad hoc wireless networks," in IEEE International Conference, Parallel Processing, Sept. 2001.

[16] C. O. S. Butenko, X. Cheng and P. Pardalos, A New Heuristic for the Minimum Connected Dominating Set Problem on Ad Hoc Wireless Network. Kluwer Academic Publisher, 2004. 
[17] S. G. C. Schurgers, V. Tsiatsis and M. Srivastava, "Topology management for sensor networks: Exploiting latency and density," in in MOBIHOC, June 9-11 2002.

[18] J. C. H. R. Zheng and L. Sha., "Asynchronous wakeup for ad hoc networks: Theory and protocol design.," in in MOBIHOC, 2003.

[19] J. H. W. Ye and D. Estrin., "An energy-efficient mac protocol for wireless sensor networks.," in IEEE Infocom, 2002.

[20] C. S. H. Y.C. Tseng and T. Y. Hsieh., "Power-saving protocols for ieee 802.11based multi-hop ad hoc networks.," in the 21st Annual Joint Conference of the IEEE Computer and Communication Societies, 2003.

[21] N. H. Xue Yang, "A wakeup scheme for sensor networks: Achieving balance between energy saving and end-to-end delay,", in In Proceedings of the 10th IEEE Real-Time and Embedded Technology and Applications Symposium (RTAS04), 2004.

[22] ns Notes and Documentation. 2000.

[23] CMU Monarch Extension to ns.

[24] A. M. S. Banerjee, "Minimum energy paths for reliable communication in multihop wireless networks," in in MOBIHOC, June 9-11 2002.

[25] Y. S. I.F. Akyildiz, W. Su and E. Cayirci, "A survey on sensor networks," IEEE Commun. Mag., vol. 20, pp. 102-114, August 2002.

[26] A. B. McDonald and T. F. Znati, "A mobility-based framework for adaptive clustering in wireless ad hoc networks," in IEEE J. Select. Areas Commun., vol. 17, pp. 1466-1487, Aug. 1999. 
[27] L. C. Z. C. Guo and J. M. Rabaey, "Low power distributed mac for ad hoc sensor radio networks.," in IEEE Globe-Com, Nov. 2001. 


\title{
VITA
}

\author{
Shariful Hasan Shaikot \\ Candidate for the Degree of \\ Master of Science
}

\section{Thesis: ENERGY AWARE ROUTING IN HIGH CAPACITY OVERLAYS IN WIRELESS SENSOR NETWORKS}

Major Field: Computer Science

Biographical:

Personal Data: Born in Dhaka, Bangladesh on 10th January, 1982.

Education:

Received the B.Sc. degree from Islamic University of Technology, Dhaka, BANGLADESH, 2002, in Computer Science \& Information Technology. Completed the requirements for the degree of Master of Science with a major in Computer Science at Oklahoma State University in May, 2007.

Experience:

Oklahoma State University

Graduate Research Assistant, Department of Agricultural Economics APR 2006 - MAY 2007

Teaching Assistant, Department of Computer Science

JAN 2005 - DEC 2005

Computer Lab Assistant, IT Division, Oklahoma State University SEP 2004 - APR 2006

Asian University of Bangladesh, Dhaka, Bangladesh

Lecturer - Department of Computer Science \& Engineering NOV 2002 - AUG 2004

Asian University Web site Supervisor \& Administrator JAN 2003 - AUG 2004

Professional Memberships: Phi Kappa Phi Honor Society 
Name: Shariful Hasan Shaikot

Institution: Oklahoma State University
Date of Degree: May, 2007

Location: Stillwater, Oklahoma

\section{Title of Study: ENERGY AWARE ROUTING IN HIGH CAPACITY OVERLAYS IN WIRELESS SENSOR NETWORKS}

Pages in Study: 25

Candidate for the Degree of Master of Science

Major Field: Computer Science

One of the most critical issues in wireless sensor networks is the limited availability of energy within the network nodes. Recently, the idea of deploying a high capacity overlay using virtual sinks with long range 802.11 links to ease congestion in the underlying sensor network has been explored. Since the VSs are battery powered, it is important to conserve energy in them too. To reduce the energy consumption, usually the shortest path (SP) route is preferred in networks that do not use topology control. However, if only a few of the VS nodes are sending data, routing along the SP may require some additional VS nodes to be turned on just for the relaying purpose which otherwise could be turned off. Since the link bandwidth is high in 802.11 (Mbps) and the sensory data generation rate is low (Kbps) in comparison to the bandwidth, a high idle-mode energy cost may be incurred in the relaying VS nodes. In this paper, we explore the idea of using minimum connected dominating set (MCDS) based routes, since more energy can be saved by switching the non-dominator VSs to sleep mode and by funnelling all the data through the MCDS nodes. We propose an energy-aware routing scheme that considers both the SP route and the MCDS nodes to discover a path along the VS network to the physical sink. Performance evaluation of the routing scheme shows a notable reduction in the overall energy consumption in the network with respect to SP routing while simultaneously maintaining an acceptable packet delivery rate.

ADVISOR'S APPROVAL: Venkatesh Sarangan 